\title{
Single mode stimulated emission from prismlike gallium nitride submicron cavities
}

\author{
C.-M. Lai \\ Department of Electronic Engineering, Ming Chuan University, Taoyuan, Taiwan 333, Republic of China \\ H.-M. Wu, P.-C. Huang, S.-L. Wang, and L.-H. Peng ${ }^{\text {a) }}$ \\ Department of Electrical Engineering, National Taiwan University, Taipei, Taiwan 106, Republic of China \\ and Institute of Electro-Optical Engineering, National Taiwan University, Taipei, Taiwan 106, \\ Republic of China
}

(Received 14 December 2006; accepted 8 March 2007; published online 5 April 2007)

\begin{abstract}
The authors report single mode stimulated emission from optical pumping of prismlike gallium nitride $(\mathrm{GaN})$ with a side length of $0.75 \mu \mathrm{m}$. The cavities were formed by reaction-rate-limited photoetching that preserved the nonpolar $\{10 \overline{1} 0\}$ or $\{11 \overline{2} 0\}$ facets. They were characterized by an average quality factor above $10^{3}$ and an equivalent facet reflectivity exceeding $98 \%$, which allowed field amplification by repeated internal reflections in the transverse plane and field polarization along the $c$ axis. Slight spectral blueshift $(0.35 \mathrm{~nm})$ and narrowing in linewidth $(\sim 0.4 \mathrm{~nm})$ were observed with increase of pump intensity. These observations manifested resonant coupling of the band edge emission to a single mode of the prismlike GaN cavity. (C) 2007 American Institute of Physics. [DOI: 10.1063/1.2720259]
\end{abstract}

Semiconductor nanocrystals provide mode confinement at a characteristic length comparable to an optical wavelength and have emerged as a versatile building block to construct photonic circuitry. ${ }^{1}$ Such structures can act not only as a passive waveguide or resonator to direct the wave propagation but also as an active medium to control light emission. ${ }^{2}$ The evanescent waves along the nanocrystals can further provide a sensor function for biochemical detection. ${ }^{3}$ These unique properties motivate research activities in material synthesis of high crystalline nanocrystals. Recent reports on optically/electrically pumped nanowire lasers of gallium nitride $(\mathrm{GaN}),{ }^{4,5}$ zinc oxide $(\mathrm{ZnO}),{ }^{6}$ and cadmium sulfide (CdS) (Ref. 7) represent one such progress in the nanocrystal research. The characteristics of these nanowire lasers can be ascribed to those of electron-hole plasma and multilongitudinal modes of a one-dimensional Fabry-Pérot cavity. These experiments inspire theoretical investigation to clarify the effects of mode reflectivity, ${ }^{8}$ gain anisotropy, ${ }^{9}$ and band gap renormalization ${ }^{10}$ on the lasing mechanism. An important revelation of these analyses is that the facet reflectivity exhibits a complicated dependence on the polarization of the cavity mode and the size of the nanowires. The facet reflectivity of nanocrystals can therefore deviate significantly from that predicted by the classical Fresnel formulation. A low value of facet reflectivity $(R)$ can further affect the cavity quality $(Q)$ factor and increase the threshold gain $\left(G_{\mathrm{th}}\right)$ of nanowire laser of length $L$ since $Q=-2 L k_{z} / \ln \left|R_{1} R_{2}\right|$ and $G_{\mathrm{th}}=-(2 L)^{-1} \ln \left|R_{1} R_{2}\right|^{8}$

A strategy to increase the cavity $Q$ factor is to include a photonic band gap structure to control the facet reflectivity. A typical $Q$ factor exceeding $10^{3}$ can be realized in conventional III-V and II-VI devices built with a microcavity or Bragg reflector. ${ }^{11}$ For the GaN emitter, however, only moderate $(\sim 300)$ or low $(\sim 185)$ value of the $Q$ factor was observed on a thin membrane ${ }^{12}$ or bulk GaN (Ref. 13) when

\footnotetext{
${ }^{a)}$ Electronic mail: peng@cc.ee.ntu.edu.tw
}

photonic crystal structures were added. It has been known that the deterioration of $Q$ factor is due to the issues of material damage and imperfect structures. ${ }^{14}$ For example, dry etching of $\mathrm{GaN}$ can result in a rough surface on the circumference $^{15}$ or a preferential loss of the nitrogen element ${ }^{16}$ which would require sophisticated postetch annealing to heal. ${ }^{17}$ Theoretical analysis also indicates that geometric imperfections such as wavy boundaries, inclined sidewalls, and rounded corners can significantly reduce the cavity $Q$ factor from the value predicted for an ideal cavity structure. $^{18}$

These observations prompt a need to develop alternative methods such that high- $Q$ photonic devices can be fabricated on GaN. In this regard, the use of whispering gallery (WG)like cavity modes to provide repeated internal reflections represents a promising route to expand the free spectral range and to achieve a single mode emission. ${ }^{19}$ This approach has been applied to the indium gallium arsenide phosphide (InGaAsP) material system and has resulted in the operation of high- $Q\left(>10^{3}\right)$ low-threshold microdisk lasers. ${ }^{20,21}$ In this letter, we report a method that uses self-assembled photomask and crystallographic photoenhanced chemical (PEC) wet etching to realize prismlike GaN submicron laser cavities. Stimulated emission at GaN band edge, in resonance with a single mode of the prismlike cavity, was observed with the field polarized along the crystal $c$ axis.

Our device fabrication began with forming a twodimensional (2D) distribution of close-packed platinum (Pt)/ titanium (Ti) pads of triangular shape on the $+c$ plane of $n^{-}$ doped GaN. This was achieved by using metal lift-off from a monolayer photomask made of self-assembled polystyrene spheres. ${ }^{22}$ To perform the PEC reaction of GaN, a galvanic cell consisting of a patterned GaN electrode described above and a Pt counterelectrode were immersed into a potassium hydroxide $(\mathrm{KOH})$ electrolyte of $p \mathrm{H}=11.7$ and illuminated with a $254 \mathrm{~nm}$ mercury light source at an intensity of $\sim 5 \mathrm{~mW} / \mathrm{cm}^{2}$. In the PEC process, the $2 \mathrm{D} \mathrm{Pt} / \mathrm{Ti}$ metallic pads served as the local cathode and the unmasked region of 


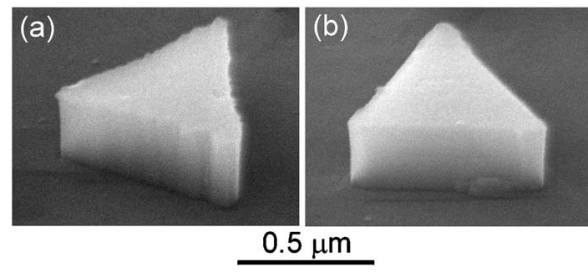

FIG. 1. Magnified SEM micrographs showing GaN prismlike cavities with a side length of $\sim 0.70 \mu \mathrm{m}$ and bounded by optically smooth (a) $\{11 \overline{2} 0\}$ and (b) $\{10 \overline{1} 0\}$ facets (with Pt/Ti metal mask on top).

the GaN as the local anode. Our studies indicate that the PEC oxidation and etching of $\mathrm{GaN}$ take place due to oxidative dissolution of GaN with the photogenerated carriers. ${ }^{23,24}$ Surface band bending of the lightly or $n$-type doped $\mathrm{GaN}$ (Ref. 25) can activate the PEC reaction without the need of an external electric bias. The PEC processing of GaN, therefore, can retain the material's stoichiometry. ${ }^{26}$ Prior to taking images of the etch patterns, the samples were briefly immersed in $200{ }^{\circ} \mathrm{C}$ molten $\mathrm{KOH}$ to remove the residual whiskerlike material defects which were sparsely left in the local anodes after the PEC process. ${ }^{27}$

Figure 1 showed the scanning electron microscope (SEM) pictures taken from a GaN sample that has undergone a process of 15 min of PEC etching and $20 \mathrm{~s}$ of molten $\mathrm{KOH}$ treatment. Prismlike cavities with a side length as small as $0.70 \mu \mathrm{m}$ and sharp/straight boundaries are clearly discernable in Fig. 1. Close examination of the magnified SEM micrographs indicated that such cavities were bounded by optically smooth $\{11 \overline{2} 0\}_{\mathrm{GaN}}$ or $\{10 \overline{1} 0\}_{\mathrm{GaN}}$ facets. These observations signify the importance of using closely spaced electrodes to activate reaction-rate-limited PEC processing. As a result, one can preserve the nonpolar $\{11 \overline{2} 0\}$ or $\{10 \overline{1} 0\}$ facets to form prismlike cavities due to a vanishing surface field effect on the PEC reaction. ${ }^{28}$

Optical characterization of the GaN submicron cavities was performed by using a microphotoluminescence ( $\mu$-PL) setup. The optical excitation was the fourth harmonic $(266 \mathrm{~nm})$ of a Nd:YAG (yttrium aluminum garnet) laser of $500 \mathrm{ps}$ pulse width focused to a $5 \mu \mathrm{m}$ diameter spot size. We used a different set of $\mathrm{GaN}$ samples with a side length of $\sim 0.75 \mu \mathrm{m}$ for the optical characterization. The roomtemperature luminescent signals were collected from the side of the sample by micro-fiber-optics positioned at $\sim 85^{\circ}$ to the sample normal, as schematically drawn in the inset of Fig. 3 . The data were dispersed via a grating spectrometer and analyzed with a linear array of charge coupled devices. The overall system spectral resolution was $\sim 0.1 \mathrm{~nm}$.

Illustrated in Fig. 2 are the $\mu$-PL spectra taken from a planar $\mathrm{GaN}$ (sample A) of $4 \mu \mathrm{m}$ thickness and from the prismlike GaN (samples B and C) which had a nominal side length/cavity height of $0.75 / 0.5 \mu \mathrm{m}$ but different $\mathrm{GaN}$ epilayer thicknesses of 4 and $0.8 \mu \mathrm{m}$, respectively. Here the $266 \mathrm{~nm}$ pump laser intensity was fixed at $\sim 1.5 \mathrm{MW} / \mathrm{cm}^{2}$ [which is below the "soft turn-on" intensity of $2 \mathrm{MW} / \mathrm{cm}^{2}$ referred to Fig. 3(a)]. We noted a characteristic spectral change from (a) broad band edge emission [full width at half maximum (FWHM) $\delta \lambda \sim 14 \mathrm{~nm}$ ] centered at $365 \mathrm{~nm}$ for planar GaN sample $A$ to a sharp single resonant peak $(\delta \lambda=0.34 / 0.29 \mathrm{~nm}$ for sample B/C) located either at the (b) shorter (364 nm, sample B) or (c) longer $(370 \mathrm{~nm}$, sample C) wavelength side of the emission spectrum. A cavity $Q$ Downloaded 09 Feb 2009 to 140.112.113.225. Redistribution subject

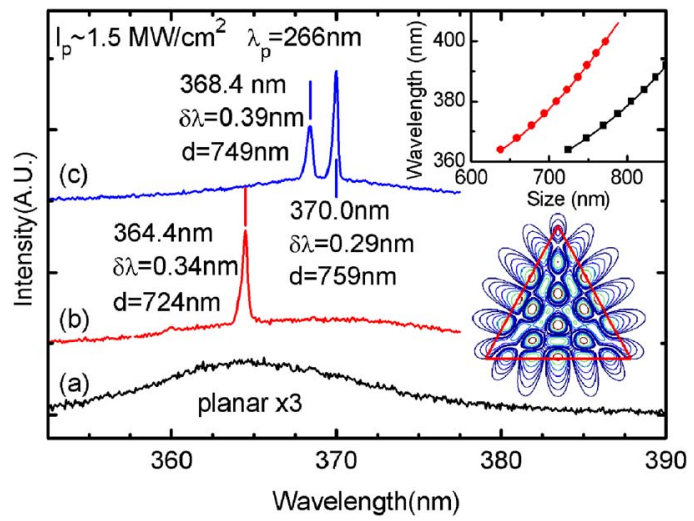

FIG. 2. (Color online) $\mu$-PL spectra of (a) planar sample A and prismlike GaN samples $B$ and $C$ having a nominal side length/prism height of $0.75 / 0.5 \mu \mathrm{m}$ but different GaN layer thicknesses of (b) $4 \mu \mathrm{m}$ and (c) $0.8 \mu \mathrm{m}$. Inset: calculated field intensity and wavelength of the cavity modes as a function of the cavity side length.

factor exceeding $10^{3}$ can be derived for the prismlike $\mathrm{GaN}$ samples B and C. This value is comparable to those ( $Q$ of 500-1300) reported for state-of-the-art $\mathrm{GaN}$ nanowire lasers ${ }^{4,5}$ and is one order of magnitude superior to that $(Q \sim 80)$ of $\mathrm{GaN}$ microdisks ( $\sim 20 \mu \mathrm{m}$ diameter $)$ fabricated by the dry etching method. ${ }^{29}$ In addition, light emission (data not shown) from the prismlike cavity was found to be polarized along the crystal $c$ axis (i.e., TM polarized), as measured in a side-collection configuration. Only a broad emission from bulk GaN was detected when measured from the sample normal. These results suggest that the observed narrow-band emission can be modeled as due to the transverse mode of a quasi-2D optical system. Using finitedifference time-domain (FDTD) analysis, ${ }^{30}$ one can utilize the cavity side length $d$ and the material dispersion ${ }^{31}$ as the input parameters to evaluate the cavity mode pattern and $Q$ factor.

Our calculation, as shown in the inset of Fig. 2, indicates that the change of cavity emission wavelength $\lambda$ in the prismlike GaN samples B and C was due to a sensitive dependence of the cavity phase-matching condition on the prism side length $d .{ }^{18}$ Associated with the degeneracy of the cavity mode energy we found two distinct field patterns. They can be understood as being transformed from the clockwise and counterclockwise components of the WG

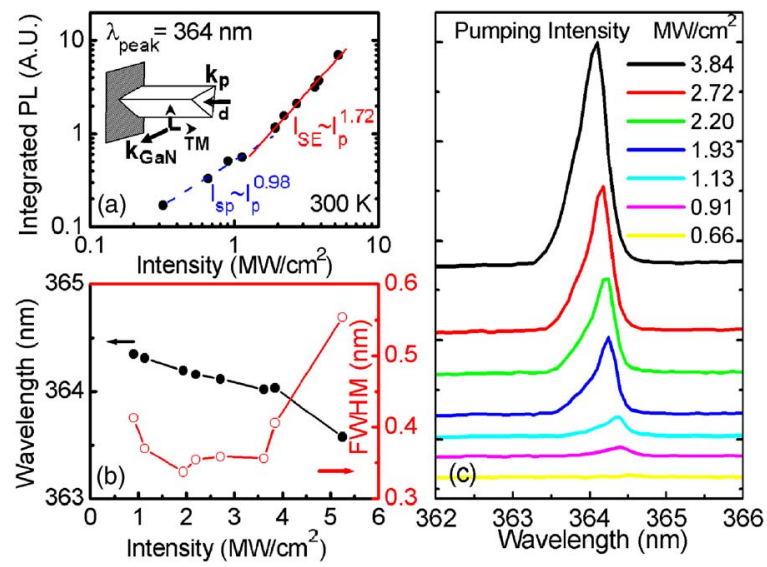

FIG. 3. (Color online) (a) Integrated PL intensity, (b) peak wavelength and FWHM, and (c) emission spectra of sample B as a function of pump intensity.

to AIP license or copyright; see http://apl.aip.org/apl/copyright.jsp 
modes of a microdisk as the cavity shape changes from circular to triangular. Shown at $3 \mathrm{~dB}$ intervals in Fig. 2 was the calculated field intensity for one of such degenerate cavity modes that partially preserves the $\sigma_{v}$ reflection symmetry of the prismlike cavity. The calculated $Q$ factor of 1308 for a $759 \mathrm{~nm}$ size GaN cavity emitting at $\lambda=370 \mathrm{~nm}$ matched that of the experimental data $(Q=1275)$ as inferred from Fig. 2(c). The $Q$ value can also be estimated from semiclassical analysis by using $Q=Q_{0}[1-(\lambda / n) \partial n / \partial \lambda]$, where the material dispersion $\partial n / \partial \lambda$ enhances the cavity $Q$ factor near the band edge.

A subtlety arising from our analysis was an occurrence of a large free spectral range ( $\geqslant 20 \mathrm{~nm}$ ) between two consecutive cavity modes, as shown in Fig. 2. Here we note a typical GaN gain spectrum under an excitation condition comparable to that used in Figs. 2 and 3 covers a finite spectral width from 355 to $370 \mathrm{~nm} .{ }^{32}$ By designing the cavity size to allow only the short wavelength cavity mode to overlap with the gain spectrum but exclude the long wavelength counterpart, one can ensure the $\mathrm{GaN}$ emission to be resonant with a single cavity mode. From semiclassical analysis one can further relate the measured $Q$ factor to an equivalent facet reflectivity $R$ of a triangular cavity according to $Q=\left[3 \pi \sin (2 \pi / 3) R^{3 / 4} n d\right] /\left[\sqrt{3} \lambda\left(1-R^{3 / 2}\right)\right] .{ }^{19}$ With an average cavity size of $0.75 \mu \mathrm{m}$ and a $Q$ factor above $10^{3}$ as those shown in Fig. 2, our calculation indicated an equivalent facet reflectivity exceeding $98 \%$ for samples B and C.

Further displayed in Fig. 3 were the changes in the characteristics of emission spectra of sample B at various pump intensities. We first noted a soft turn-on of the integrated PL intensity in Fig. 3(a) and a slope change from linear to superlinear as the pump intensity was increased from below to above a threshold $I_{p}$ of $\sim 2 \mathrm{MW} / \mathrm{cm}^{2}$. They correspond to light emission from nanocrystals in the spontaneous and the stimulated emission regimes, ${ }^{5}$ and are in agreement with the calculated results for the GaN nanowire laser. ${ }^{10}$ Close examination of Fig. 3(b) revealed a slight spectral blueshift with pump intensity. Similar phenomenon was also observed in the emission spectra of CdSe quantum dot when coupled with the optical modes of a microdisk cavity. This effect was ascribed to a carrier induced change in the refractive index, which modified the energy position of the cavity mode. ${ }^{33}$ Our data further elucidate a condition of laser wavelength clamping and matching to a single cavity mode due to emission from saturated inversion, which is reminiscent of a textbook example taught on single mode laser action. ${ }^{34}$ We also noted there was a little variation in the narrow laser linewidth $\left(\mathrm{FWHM} \sim 0.4 \mathrm{~nm}\right.$ ) at pump intensity up to $I_{p}$ $=4 \mathrm{MW} / \mathrm{cm}^{2}$, whereas broadening of the laser mode occurred at higher pump intensity. They suggest a sophisticated phenomenon due to relaxation of phase fluctuations and changes in the gain spectrum and complex refractive index at higher carrier density. ${ }^{35}$

In summary, we have reported a method of using selfassembled photomask and crystallographic PEC wet etching to fabricate prismlike GaN laser cavities on $c$ plane of sapphire substrates. TM-polarized, single mode stimulated emission near the band edge of GaN was observed on cavities with a side length of $\sim 0.75 \mu \mathrm{m}$. These observations were ascribed to the formation of a high- $Q\left(>10^{3}\right)$ laser cavity bounded by optically smooth $\{10 \overline{1} 0\}$ or $\{11 \overline{2} 0\}$ GaN facets as a result of reaction-rate-limited PEC processing.

The authors acknowledge discussion with A. H. Kung at Academia Sinica. This research was supported by the National Science Council, Grant Nos. NSC 95-2221-E-002-377, NSC 95-2112-M-130-001, NSC 95-2120-M-001-006, and NSC 95-2120-M-001-009.

${ }^{1}$ R. Agarwal and C. M. Lieber, Appl. Phys. A: Mater. Sci. Process. 85, 209 (2006).

${ }^{2}$ A. V. Maslov, M. I. Bakunov, and C. Z. Ning, J. Appl. Phys. 99, 024314 (2006).

${ }^{3}$ J. Wiersig, Phys. Rev. A 67, 023807 (2003).

${ }^{4}$ S. Gradečak, F. Qian, Y. Li, H.-G. Park, and C. M. Lieber, Appl. Phys. Lett. 87, 173111 (2005).

${ }^{5}$ P. J. Pauzauskie, D. J. Sirbuly, and P. Yang, Phys. Rev. Lett. 96, 143903 (2006).

${ }^{6}$ M. H. Huang, S. Mao, H. Feick, H. Yan, Y. Wu, H. Kind, E. Weber, R. Russo, and P. Yang, Science 292, 1897 (2001).

${ }^{7}$ X. Duan, Y. Huang, R. Agarwal, and C. M. Lieber, Nature (London) 421, 241 (2003).

${ }^{8}$ A. V. Maslov and C. Z. Ning, Appl. Phys. Lett. 83, 1237 (2003).

${ }^{9}$ R. Hauschild and H. Kalt, Appl. Phys. Lett. 89, 123107 (2006).

${ }^{10}$ L. Chen and E. Towe, J. Appl. Phys. 100, 044305 (2006).

${ }^{11}$ K. J. Vahala, Nature (London) 424, 839 (2003).

${ }^{12}$ C. Meier, K. Hennessy, E. D. Haberer, R. Sharma, Y.-S. Choi, K. McGroddy, S. Keller, S. P. DenBaars, S. Nakamura, and E. L. Hu, Appl. Phys. Lett. 88, 031111 (2006).

${ }^{13}$ L.-M. Chang, C.-H. Hou, Y.-C. Ting, C.-C. Chen, C.-L. Hsu, J.-Y. Chang, C.-C. Lee, G.-T. Chen, and J.-I. Chyi, Appl. Phys. Lett. 89, 071116 (2006).

${ }^{14}$ S. Chang, N. B. Rex, R. K. Chang, G. Chong, and L. J. Guido, Appl. Phys. Lett. 75, 166 (1999).

${ }^{15}$ K. C. Zeng, L. Dai, J. Y. Lin, and H. X. Jiang, Appl. Phys. Lett. 75, 2563 (1999).

${ }^{16}$ K. J. Choi, H. W. Jang, and J.-L. Lee, Appl. Phys. Lett. 82, 1233 (2003).

${ }^{17}$ S. Keller, C. Schaake, N. A. Fichtenbaum, C. J. Neufeld, Y. Wu, K. McGroddy, A. David, S. P. DenBaars, C. Weisbuch, J. S. Speck, and U. K. Mishra, J. Appl. Phys. 100, 054314 (2006).

${ }^{18}$ S. V. Boriskina, T. M. Benson, P. Sewell, and A. I. Nosich, IEEE J. Quantum Electron. 41, 857 (2005).

${ }^{19}$ A. K. Bhowmik, Appl. Opt. 39, 3071 (2000).

${ }^{20}$ S. L. McCall, A. F. J. Levi, R. E. Slusher, S. J. Pearton, and R. A. Logan, Appl. Phys. Lett. 60, 289 (1992).

${ }^{21}$ M. Fujita, A. Sakai, and T. Baba, IEEE J. Sel. Top. Quantum Electron. 5, 673 (1999).

${ }^{22}$ J. C. Hulteen, D. A. Treichel, M. T. Smith, M. L. Duval, T. R. Jensen, and R. P. Van Duyne, J. Phys. Chem. B 103, 3854 (1999).

${ }^{23}$ L.-H. Peng, C.-H. Liao, Y.-C. Hsu, C.-S. Jong, C.-N. Huang, J.-K. Ho, C.-C. Chiu, and C.-Y. Chen, Appl. Phys. Lett. 76, 511 (2000).

${ }^{24}$ L.-H. Peng, C.-W. Chuang, J.-K. Ho, C.-N. Huang, and C.-Y. Chen, Appl. Phys. Lett. 72, 939 (1998).

${ }^{25}$ L.-H. Peng, C.-W. Shih, C.-M. Lai, C.-C. Chuo, and J.-I. Chyi, Appl. Phys. Lett. 82, 4268 (2003).

${ }^{26}$ M. S. Minsky, M. White, and E. L. Hu, Appl. Phys. Lett. 68, 1531 (1996).

${ }^{27}$ C. Youtsey, L. T. Romano, and I. Adesida, Appl. Phys. Lett. 73, 797 (1998).

${ }^{28}$ L. H. Peng, C.-Y. Lu, W.-H. Wu, and S.-L. Wang, Appl. Phys. Lett. 87, 161902 (2005).

${ }^{29}$ H. W. Choi, K. N. Hui, P. T. Lai, P. Chen, X. H. Zhang, S. Tripathy, J. H. Teng, and S. J. Chua, Appl. Phys. Lett. 89, 211101 (2006).

${ }^{30}$ S. Dey and R. Mittra, IEEE Microw. Guid. Wave Lett. 8, 415 (1998).

${ }^{31}$ N. Antoine-Vincent, F. Natali, M. Mihailovic, A. Vasson, J. Leymarie, P. Disseix, D. Byrne, F. Semond, and J. Massies, J. Appl. Phys. 93, 5222 (2003).

${ }^{32}$ J. Mickevičius, G. Tamulaitis, M. S. Shur, Q. Fareed, J. P. Zhang, and R. Gaska, J. Appl. Phys. 99, 103513 (2006).

${ }^{33}$ J. Renner, L. Worschech, A. Forchel, S. Mahapatra, and K. Brunner, Appl. Phys. Lett. 89, 231104 (2006).

${ }^{34}$ also H. Haken, Light: Laser Light Dynamics (Elsevier Science, Amsterdam, 1985), 2, p. 127.

${ }^{35}$ C. H. Henry, IEEE J. Quantum Electron. 18, 259 (1982). 